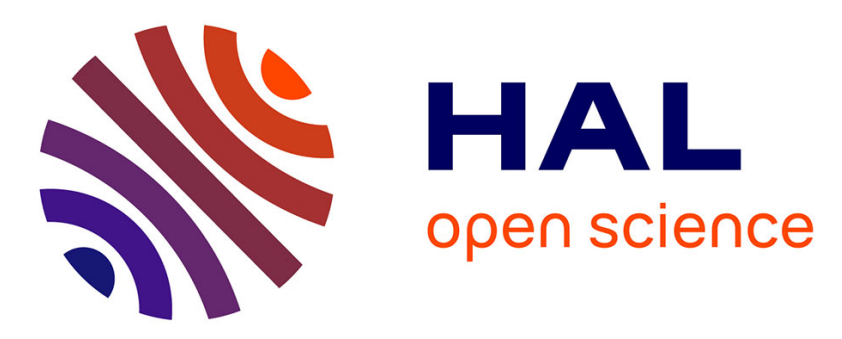

\title{
Noyens-sur-Seine, site stratifié en milieu fluviatile: une étude multidisciplinaire intégrée
}

M-C Marinval-Vigne, Daniel Mordant, G Auboire, Anne Augereau, Salvador

Bailon, C Dauphin, G Delibrias, Vincent Krier, A-S Leclerc, Chantal Leroyer, et al.

\section{To cite this version:}

M-C Marinval-Vigne, Daniel Mordant, G Auboire, Anne Augereau, Salvador Bailon, et al.. Noyenssur-Seine, site stratifié en milieu fluviatile: une étude multidisciplinaire intégrée. Bulletin de la Société préhistorique française, 1989, Du terrain au laboratoire: Pour un meilleur dialogue en archéologie, 86 (10-12), pp.370 - 379. 10.3406/bspf.1989.9894 . mnhn-03262157

\section{HAL Id: mnhn-03262157}

\section{https://hal-mnhn.archives-ouvertes.fr/mnhn-03262157}

Submitted on 16 Jun 2021

HAL is a multi-disciplinary open access archive for the deposit and dissemination of scientific research documents, whether they are published or not. The documents may come from teaching and research institutions in France or abroad, or from public or private research centers.
L'archive ouverte pluridisciplinaire HAL, est destinée au dépôt et à la diffusion de documents scientifiques de niveau recherche, publiés ou non, émanant des établissements d'enseignement et de recherche français ou étrangers, des laboratoires publics ou privés. 


\section{Noyens-sur-Seine, site stratifié en milieu fluviatile : une étude} multidisciplinaire intégrée

M.-C. Marinval-Vigne, Daniel Mordant, G. Auboire, Anne Augereau, S. Bailon, C. Dauphin, G. Delibrias, Vincent Krier, A.-S. Leclerc, Chantal Leroyer, P. Marinval, Claude Mordant, P. Rodriguez, Philippe Vilette, Jean-Daniel Vigne

\section{Citer ce document / Cite this document :}

Marinval-Vigne M.-C., Mordant Daniel, Auboire G., Augereau Anne, Bailon S., Dauphin C., Delibrias G., Krier Vincent, Leclerc A.-S., Leroyer Chantal, Marinval P., Mordant Claude, Rodriguez P., Vilette Philippe, Vigne Jean-Daniel. Noyens-sur-Seine, site stratifié en milieu fluviatile : une étude multidisciplinaire intégrée. In: Bulletin de la Société préhistorique française, tome 86 , n¹0-12, 1989. Du terrain au laboratoire : Pour un meilleur dialogue en archéologie. pp. 370-379;

doi : 10.3406/bspf.1989.9894

http://www.persee.fr/doc/bspf_0249-7638_1989_hos_86_10_9894

Document généré le 09/04/2016 


\author{
par M.-C. Marinval-Vigne, D. Mordant, G. Auboire, A. Augereau, S. Bailon, C. Dauphin, \\ G. Delibrias, V. Krier, A.-S. Leclerc, C. Leroyer, P. Marinval, C. Mordant, P. Rodriguez, \\ P. Vilette, J.-D. Vigne
}

Les fouilles les plus récentes du site de Noyen-surSeine (Seine-et-Marne) (1982-1988) ont révélé un gisement unique pour le Nord de la France. Il s'agit de dépôts tourbeux fluviatiles qui ont livré un matériel organique parfois exceptionnel (pirogue, sparteries), en excellent état de conservation, inclus dans une série stratifiée s'étendant du Préboréal au Subatlantique, du Mésolithique à l'Age du Fer (Mordant et Mordant, 1987). Plusieurs études environnementales ont été menées au fur et à mesure de la fouille et postérieurement à celles-ci. Les données les plus novatrices se rapportent au Mésolithique, durant lequel le site a connu plusieurs occupations temporaires ou non sans que cela implique nécessairement qu'il ait été un habitat permanent. Une part importante de l'intervention archéologique fut réservée aux reconstitutions paléo-environnementales et à l'étude des restes animaux et végétaux qui, en retour, permettent de jeter les bases du paléo-milieu et des comportements du groupe humain nécessaires à une meilleure compréhension de la stratégie d'exploitation des ressources, en particulier au Mésolithique.

I - ÉVOLUTION DU SYSTĖME HYDROLOGIQUE DE LA SEINE

Les études sédimentologiques, palynologiques et malacologiques ont été réalisées à partir de stations de prélèvement communes. Elles ont permis, par confrontation des résultats et en s'appuyant, pour la sédimentologie, sur les travaux de Lereton (1974) et de Frecaut et Pagney (1983), de proposer une reconstitution évolutive de l'écoulement fluvial en ce point de la vallée de la Seine depuis le Tardiglaciaire jusqu'au Subboréal. Le cadre chrono-stratigraphique est établi sur la base des datations absolues, du matériel archéologique et de l'étude palynologique. Les exemples d'études de ce type restent rares pour ces périodes, en particulier dans le centre du Bassin Parisien. Non loin de Noyen, le site de la centrale nucléaire de Nogent-sur-Seine avait toutefois déjà fourni quelques données, malheureusement très lacunaires et déconnectées de tout contexte archéologique (Delibrias et al., 1982).

\section{I.1 - Au Tardiglaciaire (coupe XV-XVI 161)}

La nappe alluviale tardiglaciaire de Noyen correspond à un système de chenaux anastomosés s'étalant largement dans la vallée. Le régime très contrasté, où alternent crues et décrues, est suffisamment puissant pour avoir creusé un lit dans la craie et avoir renouvelé le stock de granulats par des apports régionaux.

\section{I.2 - Évolution de l'écoulement au Postglaciaire}

\section{I.2.1 - Dynamique Préboréale}

Faisant suite au réseau en tresse tardiglaciaire, un système à méandres lents s'installe : des bras larges se mettent en place, en deux points éloignés du site. Ces creusements dans les graviers sont suivis d'un comblement limono-tourbeux, assez compact et riche en matière organique, significatif d'une eau courante au débit assez lent; la dynamique calme semble 
interrompue par des phases d'activité plus vive avec reprise d'érosion. Les associations polliniques mises en évidence permettent l'attribution de ces niveaux au Préboréal (coupes XVI J 236 couche 29, XXVI MN 46/47 couche 23 base).

\section{I.2.2 - Boréal : individualisation de deux secteurs}

Pour cette période, deux secteurs ont pu être individualisés : ils correspondent à deux types de dynamique alluviale évoluant indépendamment l'une de l'autre.

Après une phase d'érosion, le comblement amorcé dès le Préboréal se poursuit; le dépôt limonotourbeux, plus compact et plus organique que le précédent évoque une eau courante en régime doucement actif (coupe XV J 236, couches 29 et 25 base).

Différentes coupes témoignent de l'installation d'un système de méandres à chenaux actifs caractérisé par une évolution très rapide du cours du fleuve créant de nombreux petits bras entrecoupés. Leur mise en place est subcontemporaine. Les spectres pollinique et malacologique concordent et permettent de décrire un complexe à méandres actifs qui évoque une eau courante à dynamique relativement vive, sans développement d'une frange marécageuse. Des niveaux organiques ont été observés au sein de cet ensemble. Ils correspondent à des accumulations de débris végétaux flottés et piégés en bordure des plages internes du méandre puis scellées par l'apport d'un nouveau matériau lors de l'évolution latérale des chenaux, plutôt qu'à une sédimentation organique in situ liée à un plan d'eau dormante ou calme (coupes XV-XVI 161, couches 12-12 bis, XVI L 196 couche 22, XVI G 198 couches 21-19).

Dans le secteur de la coupe XVI L 196, on constate le creusement d'un large bras postérieur à la série des méandres actifs. Comme le prouve le dépôt organique très peu chargé en apport détritique, le chenal tend à s'isoler de la Seine active et évolue vers un bras mort. La malacofaune traduit une eau calme mais renouvelée encore de façon régulière. Le chenal semble peu soumis à des assèchements latéraux et abrite une végétation hygrophile réduite (coupes XVI L 196, couche 9, XVI J 236, couche 24).

\section{I.2.3 - L'Atlantique : marginalisation des chenaux}

Les secteurs individualisés au Boréal continuent à évoluer séparément à l'Atlantique. La tendance générale est à la marginalisation de ces chenaux.

Les coupes XVI U 211 couche 9 sup., XVI L 196 couches 8 et 7 traduisent une sédimentation en " mouille ». Une lacune d'érosion entre les couches 9 et 8 de la coupe XVI L 196, due à une reprise du courant, montre que le bras mort est encore en connexion avec le réseau actif de la Seine.

Les deux coupes témoignent cependant d'une tendance à la diminution d'activité du fleuve avec ralentissement du courant : les dépôts sont de plus en plus organiques avec peu d'éléments détritiques. Une végétation hygrophile semble s'installer dans le chenal lui-même durant le dépôt de la couche 8 .

A la fin de l'Atlantique, le chenal XVI L 196 (couches 7 et 6 ) change de nature. Le débit connaît alors un fort ralentissement et on assiste à un début d'atterrissement du bras de la rivière, ses franges s'asséchant vraisemblablement une partie de l'année.

Le chenal en phase de comblement, présentant des plans d'eau dormante, est colonisé par une végétation aquatique. Une flore terrestre se développe sur ses berges.

Bien qu'accusant une légère diminution d'activité, le chenal XXVI MN 46/47 (couche 23) est encore actif, alimenté par une eau courante peu vive.

\section{I.2.4 - Le passage de l'Atlantique au Subboréal}

Dans le secteur XVI 196, le chenal est en phase de comblement. Une baisse d'humidité liée à l'assèchement progressif du bras se marque, dans les faunes de mollusques, par le développement des espèces terrestres, et, dans le spectre pollinique, par un fort développement des graminées au détriment des hygrophiles (coupe XVI L 196, couches 5 et 4 base).

Le second chenal est toujours actif mais son régime s'affaiblit peu à peu. Des assèchements latéraux et des phases de basses eaux sont probables (coupe XXVI MN 46/47, couche 23 sup.).

\section{I.2.5 - Le Subboréal : colmatage final}

Le chenal XVI L 196 est comblé (couche 4) ; une prairie humide s'installe, comme en témoigne la prépondérance des mollusques terrestres.

Le second bras (XXVI MN 46/47, couche 4) tend à son tour à l'inactivité. Son atterrisement se poursuit mais il conserve encore un léger écoulement permanent.

\section{I.3 - Bilan chronologique (fig. 1)}

L'analyse simultanée de coupes stratigraphiques de la grande séquence de Noyen-sur-Seine par les sédimentologue, malacologue et palynologue a permis de définir l'évolution du système hydrologique de 


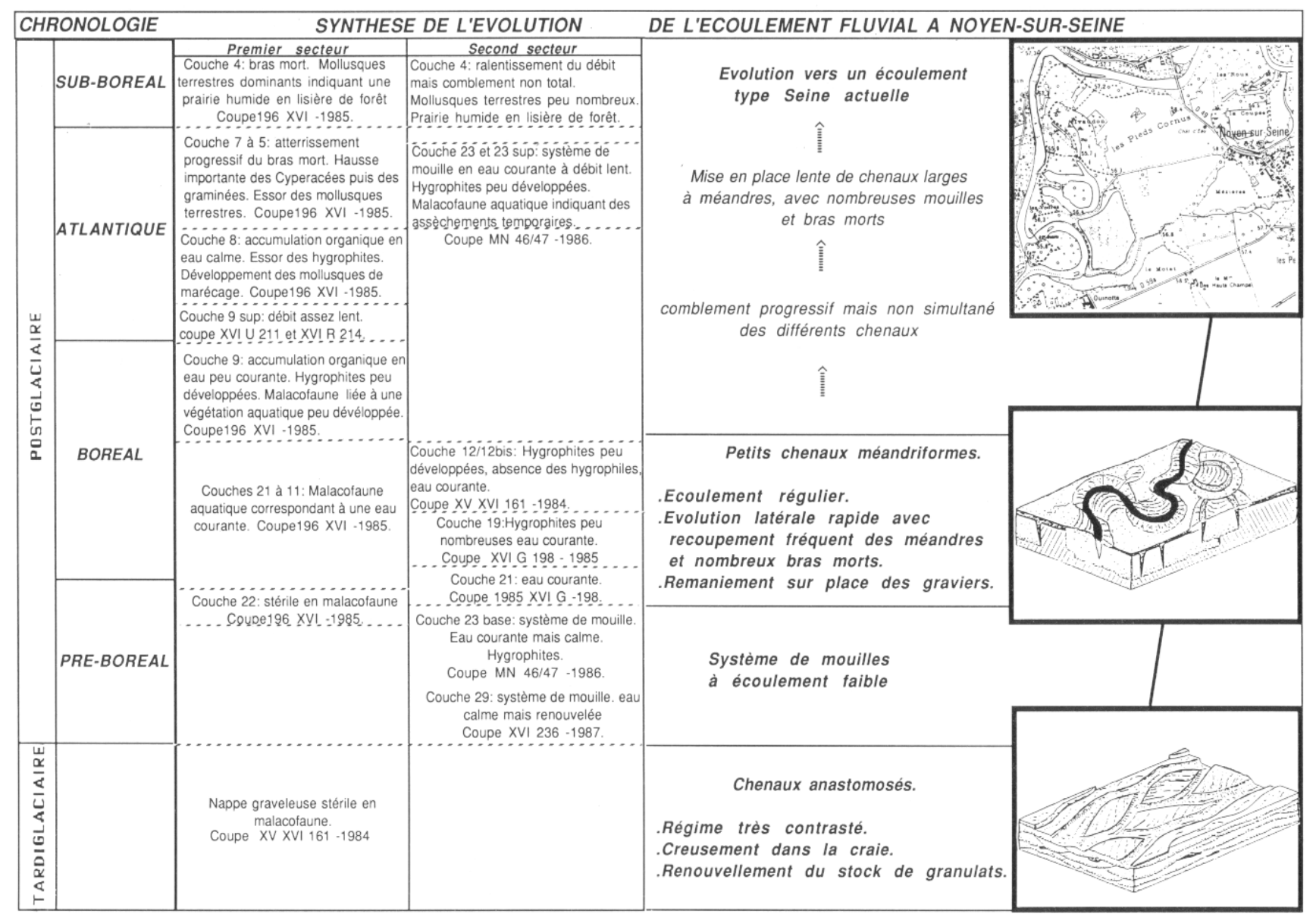

Fig. 1 - Synthèse de l'évolution de l'écoulement fluvial à Noyen-sur-Seine.

la Seine à un endroit de la "Bassée » qui semble avoir attiré l'Homme depuis 8 millénaires au moins.

La transition d'un système d'écoulement tressé en chenaux anastomosés au Tardiglaciaire vers une dynamique plus calme au Postglaciaire n'apparaît pas de façon uniforme sur l'ensemble du site. Si dans certains secteurs, un système de comblement en mouille s'instaure dès le Préboréal, dans d'autres un réseau complexe de petits chenaux méandriformes à évolution rapide perdure au Boréal, alors que les Mésolithiques fréquentent les lieux.

Peu à peu, ces chenaux s'isolent du réseau actif de la Seine par un lent atterrissement et vont constituer des bras morts. Cette tendance à l'inactivité s'observe de façon générale, mais on constate un décalage du phénomène dans le temps d'un point à un autre du site : certains chenaux sont déjà comblés tandis que d'autres conservent un écoulement permanent quoique très amoindri. Cette modification débuterait à la fin du Boréal pour s'accentuer au Subboréal (Néolithique moyen et final) où elle aboutit à un comblement complet (Bronze ancien) et à la mise en place d'un lit unique à l'image de la Seine actuelle.

\section{II. - ÉVOLUTION DE L'ENVIRONNEMENT BIOLOGIQUE}

\section{II.1 - Les végétaux}

\section{II.1.1 Apports de la palynologie}

Le diagramme pollinique synthétique et schématique de la fig. 2 retrace la dynamique de la végétation mise en évidence à Noyen par l'étude de sept séquences dégagées dans différents paléo-chenaux. Les ensembles polliniques individualisés autorisent l'établissement d'une contemporanéité entre divers niveaux et leur intégration dans le cadre chronostratigraphique du Postglaciaire.

Préboréal (ensemble $1:$ c. 29, coupe XVI J 236 et ensemble 2 : c. 29 et c. 23 base, coupe XXVI MN 46/47).

La végétation semble d'abord caractérisée par la prédominance massive du Pin, accompagné de quel- 
ques rares noisetiers et chênes. Ensuite ces deux derniers se développent progressivement au sein de cette pinède.

Boréal (ensemble $3:$ c. 29 , c. 23 base et c. 21 , coupe XVI B 199 et ensemble 4 : c. 25, coupe XV J 236 et c. 19 , couche XVI G 198 , et c. 12 et 12 bis, coupe XV-XVI V 161 et c. 9, coupe XVII).

Le Noisetier, qui devance le Chêne puis l'Orme, poursuit sa progression parallèlement au recul du Pin, jusqu'à devenir majoritaire ou tout au moins à égaler ce dernier. Le Chêne et l'Orme suivent la même évolution bien que plus faiblement, tandis qu'apparaît le Tilleul.

Atlantique ancien (ensemble $5:$ c. 9 et c. 24, coupe XV J 236 et ensemble 6: c. 9 sup., coupe XVI U 211).

Le Noisetier amorce une courbe régressive tandis que le Chêne et l'Orme se maintiennent. Bientôt ces derniers commencent eux aussi à décliner tandis que le Pin connaît un regain de réprésentation.

Atlantique récent (ensemble $7:$ c. 9 sup. et ensemble $8:$ c. 7 et 8 , coupe XVI L 196).

Le Noisetier, le Chêne et l'Orme poursuivent leur déclin, tandis que le Tilleul atteint son maximum de représentation. L'aulne, jusque-là, faiblement représenté progresse nettement.

Sub-boréal (ensemble 9 : c. 6, 5, 4, coupe XVI L 196 et C. 23, 23 sup., coupe XXVI MN 46/47).

L'aulnaie s'installe sur le site ; la forte représentation de l'Aulne masque alors celle de la forêt caducifoliée.

Il ressort de cette étude une impression de faible anthropisation du paysage. Les premières déforestations possibles ne s'enregistrent que dans un des niveaux du Néolithique moyen.

\section{II.1.2 - Apports de la carpologie}

L'étude a porté uniquement sur la couche 9, datée du Mésolithique. Les échantillons de sédiment contenaient assez peu de paléo-semences et la conservation n'est pas excellente, contrairement aux autres macro-restes végétaux. Le cortège floristique est composé exclusivement de plantes spontanées, soit aquatiques (poussant dans des eaux stagnantes ou à faible courant) soit arbustives ou arborescentes (qui devaient croître sur les berges du cours d'eau). Seize taxons ont été identifiés : le Chêne (Quercus $s p$.), le Noisetier (Corylus avellana), le Hêtre (Fagus sylvatica), la Vigne sauvage (Vitis sylvestris), l'Aubépine à un style (Crataegus monogyna), l'Iris jaune (Iris pseudocorus), le Merisier (Prunus avium), le Cornouiller sanguin (Cornus sanguinea), le Nénuphar jaune (Nuphar lutea), le Potamot nageant (Potamogeton natans), le Laîche vésiculeuse (Carex cf. vesicaria), le groupe des Renoncules aquatiques (Ranunculus aquatilis agg.), le Lycope (Lycopus europaeus), la Violette tricolore (Viola tricolor agg.), des Graminées (Poaceae) et des Composées (Asteraceae).

La question est de savoir si ces semences résultent de collectes par l'Homme ou si elles correspondent à un dépôt naturel à la suite de leur dissémination. L'absence de carporestes carbonisés et l'existence de nombreuses plantes aquatiques font plutôt pencher pour une accumulation en grande partie naturelle.

\section{II.2 - Les animaux}

Les restes de Vertébrés sont nombreux et dans un état de conservation exceptionnel. Ils se répartissent entre cinq grands ensembles chronostratigraphiques : les chenaux inférieurs (Ens. 1, Mésolithique, Préboréal au Boréal), les tourbes inférieures (Ens. 2, Mésolithique moyen, Boréal à l'Atlantique), les tourbes supérieures comportant l'ensemble 3 (Mésolithique final, Atlantique ancien), l'ensemble 4 (Néolithique ancien, Atlantique), et l'ensemble 5 (Néolithique moyen II, Sub-boréal). Les ensembles les mieux documentés sont ceux du Mésolithique (2 et 3). Les 5 groupes de Vertébrés (Poissons, Amphibiens, Reptiles, Oiseaux et Mammifères) sont attestés de façon inégale. Les plus représentés sont les Poissons et les Mammifères.

Les ossements de poissons (Dauphin, 1987) étaient concentrés dans une zone limitée du chenal. Le spectre faunique comporte presque exclusivement l'Anguille (Anguilla anguilla) et le Brochet (Esox lucius), Cyprinidés et Percidés étant réduits à de très petites quantités. Ce spectre ne peut en aucun cas correspondre à une taphocénose naturelle. De plus, la saison de mort des individus ne couvre pas l'ensemble de l'année. Enfin, dans les ensembles mésolithiques ( 2 et 3 ), de nombreux ossements sont brûlés et quelques-uns portent des traces de découpe culinaire. Il est clair que les ossements de poissons proviennent en grande majorité de l'activité humaine. Il en va de même pour ceux des grands Mammifères, qui résultent de la prise des repas au voisinage immédiat du lieu de dépôt, et même des ossements d'Oiseaux où les rares espèces aquatiques représentées portent également des traces de consommation. Même les nombreux restes de Cistude (Emys orbicularis) portent des traces de brûlures et de découpes. Il n'y a guère que les Amphibiens, d'ailleurs très peu représentés, qui pourraient témoigner de dépôts naturels.

Dans ces conditions, les données paléoenvironne- 


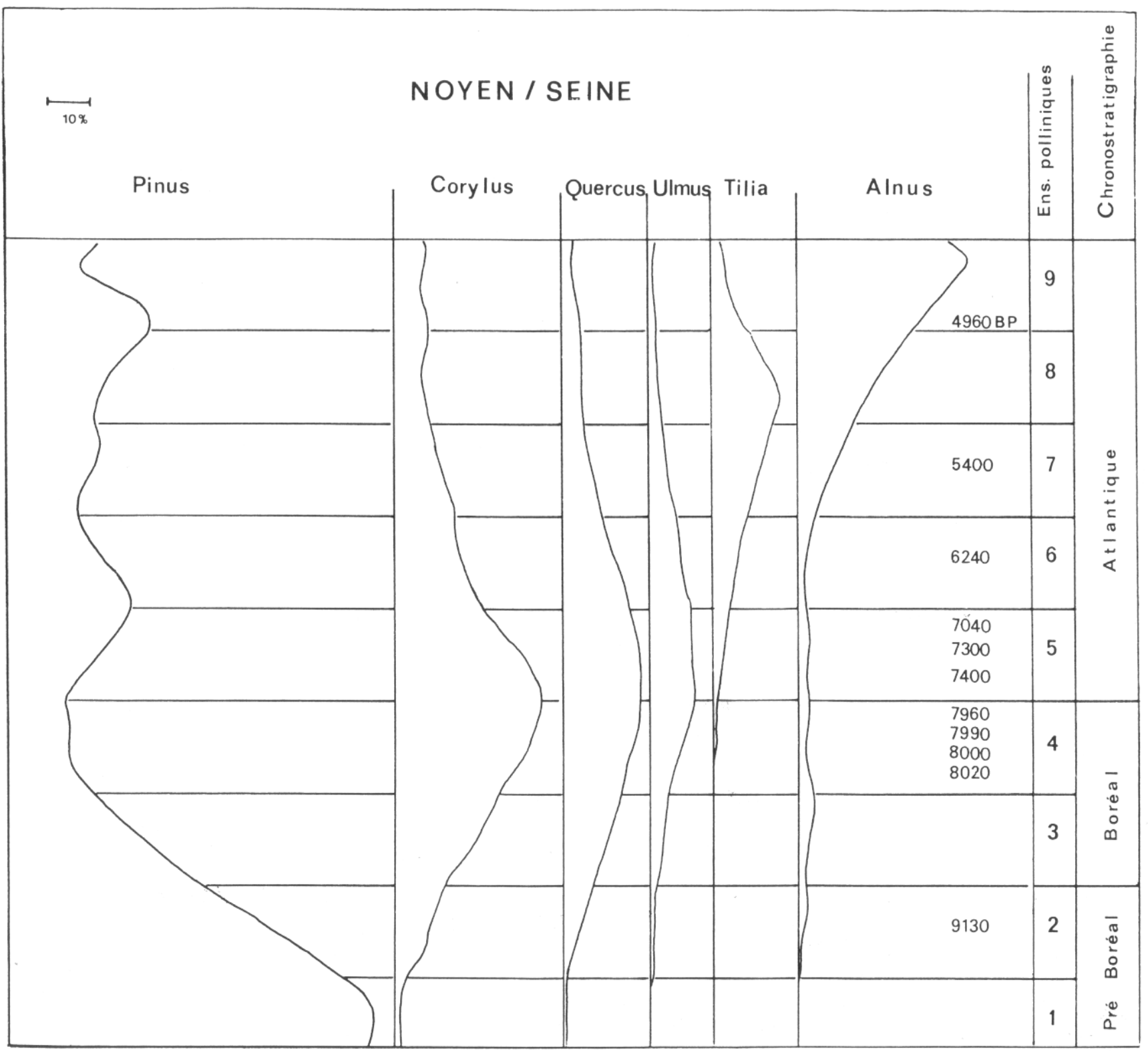

Ch. LEROYER 1989

Fig. 2 - Diagramme pollinique synthétique et simplifié de Noyen-sur-Seine

mentales issues de la faune de Vertébrés sont en partie biaisées par les choix anthropiques. Elles sont de ce fait d'une portée limitée.

On peut toutefois noter que les espèces aquatiques (le Brochet, l'Anguille, la Perche, Perca fluviatilis, la Tanche, Tinca tinca, les Grenouilles, cf. Rana, la Cistude) et celles qui sont inféodées aux milieux humides (le Crapaud commun, Bufo cf. bufo, la Couleuvre à collier, Natrix sp., les Canards, la Loutre, Lutra lutra, le Putois, Mustela putorius, le Campagnol amphibie, Arvicola cf. terrestris, le Castor, Castor fiber) ne permettent pas de distinguer d'importantes variations du micro-milieu fluviatile durant les différentes phases du Mésolithique.
Certains Oiseaux (le Geai des chênes, cf. Garrulus glandarius) et la plupart des Mammifères (l'ours, Ursus arctos, la Martre, Martres martes, le Blaireau, Meles meles, le Chat sauvage, Felis silvestris, le Lynx, Lynx lynx, le Castor, le Sanglier, Sus scrofa, le Cerf, Cervus elaphus, le Chevreuil, Capreolus capreolus) témoignent de la proximité et de l'abondance de milieux boisés durant tout le Boréal et l'Atlantique au moins. En contrepartie, les espèces de milieux ouverts sont absentes (Lièvre, Microtidés), très rares (Equus sp.) ou secondaires (Chevreuil, Aurochs, Bos primigenius) et les espèces de lisière (Renard, Vulpes vulpes, Loup, Canis lupus, Belette et Hermine...) sont peu représentées tout au long de la séquence. 
Cette relative stabilité des faunes semble s'opposer aux données géologiques, malacologiques et palynologiques. Il n'en est rien si l'on considère que la séquence chronologique décrite par les ensembles fauniques ne s'étend que de la fin du Boréal à l'Atlantique moyen, et que les espèces dont on dispose ont une sensibilité écologique bien plus faible que les végétaux et que certains mollusques.

III - L'HOMME ET LES RESSOURCES NATURELLES

\section{III.1 - L'alimentation d'origine animale}

\section{III.1.1 - La Cistude}

La consommation de la Cistude (Emys orbicularis) est largement attestée durant tout le Mésolithique et se poursuit au Néolithique. L'excellent état de conservation du matériel a permis de repérer des traces de découpe et de feu qui permettront d'évoquer les techniques de consommation de cette espèce au Mésolithique.

\section{III.1.2 - Les Poissons (Dauphin, 1987)}

L'échantillon issu des couches mésolithiques est constitué d'environ 2000 corps vertébraux et 127 pièces crâniennes.

La pêche était concentrée sur le Brochet et l'Anguille. Le premier domine dans l'ensemble 2, alors que c'est l'inverse dans le Mésolithique final, précisément là où ont été découvertes les nasses. Les traces de brûlures, visibles sur les vertèbres d'anguilles sont peut-être des indices d'une technique de fumage à feu couvert. Il semble qu'il existe une pêche plus abondante en été. Ces éléments évoquent une activité saisonnière avec une certaine part de mise en réserve, hypothèse qu'il faudra confronter aux autres données.

\section{III.1.3 - Les Oiseaux}

La plupart des oiseaux identifiés peuvent avoir été tués à la chasse. Au Mésolithique moyen, il s'agit de la Grue cendrée (Grus grus), du Canard colvert (Anas platyrhyncos), de la Foulque macroule (cf. Fulica atra) et du Geai des chênes (cf. Garrulus glandarius). Au Mésolithique final, on trouve encore le Canard colvert et le Geai des chênes, auxquels s'ajoutent le Fuligule nyroca (Aythya nyroca) et le
Garrot à œil d'or (Bucephala clangula), deux canards plongeurs hivernants en France. Les très faibles effectifs montrent que la chasse aux oiseaux constituaient un apport occasionnel. Les os ne portent aucune trace de décarnisation, ni de brûlure mais il est à noter que pour le Canard colvert, qui est l'espèce la plus fréquente, les os des extémités distales des membres sont très peu (ou pas) représentés, ce qui peut faire penser à une consommation par l'Homme.

Si l'hypothèse de chasse est retenue, on peut constater que trois espèces sur six sont migratrices (l'Europe constituant leurs aires d'hivernage) et donc la capture de ces oiseaux aurait eu lieu plutôt de la fin de l'été au début du printemps. Mais une grande prudence s'impose sur un si petit lot.

\section{III.1.4 - Les Mammifères}

Plus de 5000 restes mammaliens ont été collectés sur l'ensemble de la séquence; à ce jour, 3154 ont été déterminés (Vigne et Marinval-Vigne, à paraître). L'ensemble ostéologique le plus riche, dans un état de conservation remarquable, provient des couches mésolithiques (ensemble 2:1766 restes déterminés ; ensemble 3:1 158 restes déterminés).

Pour le Mésolithique moyen (ensemble 2), l'étude de la fréquence des parties du squelette, désigne clairement des camps de base occupés durant une grande partie de l'année, peut-être du printemps à la fin de l'automne. Les proies étaient abattues lors de courtes expéditions de chasse. Il est possible que l'homme ait été accompagné dans ses opérations de chasse par des loups en cours de domestication (Vigne et Marinval-Vigne, 1988). Débitées sur le lieu de chasse, les carcasses étaient rapportées au campement entières (Chevreuil) ou en quartiers (Sanglier, Aurochs, Cerf). Le schéma de découpe, mis en évidence grâce à l'excellente conservation des surfaces osseuses, était relativement stéréotypé. Les viandes étaient généralement grillées, et l'exploitation alimentaire de la carcasse était maximale (récupération systématique de la moelle osseuse...).

Les écosystèmes exploités sont le fleuve (petits Carnivores), les lisières (Loup, Renard, Aurochs, Chevreuil) et surtout la forêt (Cerf, Sanglier, Chat sauvage, Lynx...). Le Cerf est le gibier de prédilection $(43 \%$ du Nombre de Restes, NR, et $56 \%$ du Poids de Restes, PR ; fig. 3). Il est tué préférentiellement entre 6 et 9 ans. Puis vient le Sanglier ( $27 \%$ du NR et $11 \%$ du PR), abattu surtout dans ses deuxième et troisième années. Le Chevreuil est relativement abondant et le spectre de faune diversifié.

L'occupation du Mésolithique final (Atlantique) correspond également à un camp de base. Les 

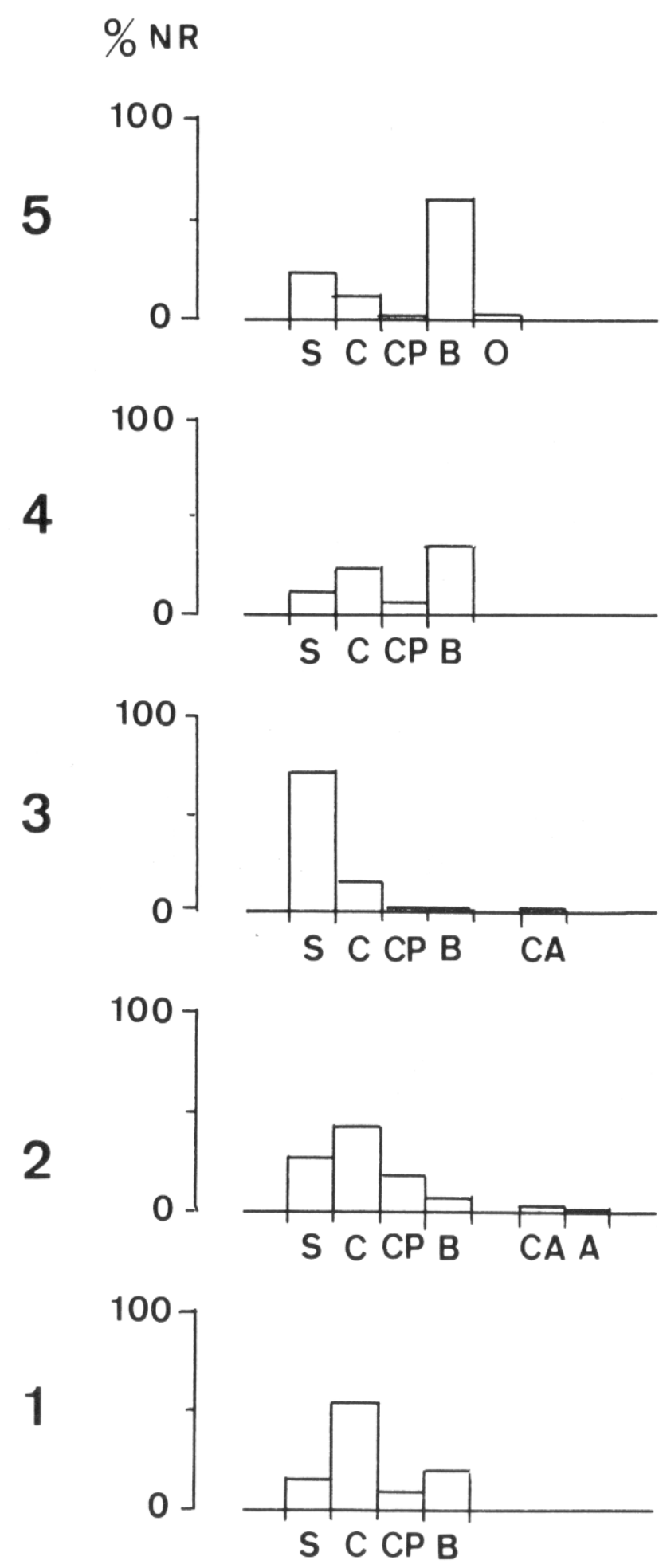

Fig. 3 - Évolution du spectre faunique des Mammifères exprimé en Nombre de Restes (NR) dans les principaux ensembles chronostratigraphiques de Noyen-sur-Seine : 1, Ensemble 1 (Mésolithique des chenaux inférieurs ; $\mathrm{NR}=77) ; 2$, Ensemble 2 (Mésolithique moyen ; NR $=2847$ ) ; 3, Ensemble 3 (Mésolithique final ; $N R=1883$ ) ; 4, Ensemble 4 (Néolithique ancien ; $N R=17$ ) ; 5 , Ensemble 5 (Néolithique moyen ; $N R=123$ ). $S$, Sus scrofa ; C, Cervus elaphus ; CP, Capreolus capreolus ; B, Bos ; O, Ovis aries ; CA, Carnivores ; A, Autres (Vigne et Marinval-Vigne, à paraître)

écosystèmes exploités sont les mêmes qu'au Boréal, mais certaines pratiques alimentaires (découpe, mise en réserve, cuisson...) sont très différentes de celles observées au Mésolithique moyen, et le spectre faunique est réduit à deux espèces principales (Cerf et Sanglier, fig. 3). Le Sanglier, dont la taille est semblable à celle observée dans le Mésolithique moyen, représente $70 \%$ du NR et $49 \%$ du PR. Pour cette espèce, on note deux pics d'abattage, aux environs de 7-8 mois et après $10-12$ mois. Ils pourraient résulter d'une chasse opportuniste à un moment de l'année où seules ces deux classes de jeunes étaient présentes, hypothèse corroborée par tous les autres indices de saisonnalité. Tout cela évoque une occupation limitée dans le temps (quelques jours?), probablement à la fin de l'été, par un groupe humain culturellement différent de celui du Mésolithique moyen.

Il apparaît toutefois nettement que ces Mésolithiques n'étaient pas sur le chemin d'une néolithisation. Le système d'approvisionnement reste typiquement mésolithique, et on note une absence presque totale de contacts avec les Néolithiques déjà implantés dans la vallée à cette époque (D. Mordant, comm. pers.). Tout au plus peut-on supposer (avec la plus grande prudence) que quelques chiens venus des villages néolithiques ont erré sur les restes du campement des Mésolithiques, ou que ces derniers ont occasionnellement chassé (ou échangé ?) des bovins échappés aux Néolithiques, comme pourrait l'indiquer un très petit nombre de restes de taille réduite (Bos cf. taurus ?). Mais l'absence totale d'os de Mouton traduit bien l'étanchéité des frontières socio-économiques qui séparaient les deux groupes.

Ce n'est qu'avec l'ensemble 4 qu'on trouve le premier spectre faunique de type néolithique (fig. 3), dominé par des bovins de grande taille, mais pour la plupart domestiques. Dans l'ensemble 5, l'alimentation est dominée par le Bœuf (Bos taurus).

\section{III.2 - L'industrie et l'artisanat}

\section{III.2.1 - L'industrie lithique (fig. 4)}

L'industrie en silex des couches $7 d, 9$ et $10 \mathrm{a}$ se caractérise par une production sur éclat façonnée dans un silex local (silex crétacé). Les pièces retouchées sont essentiellement représentées par des outils du fonds commun : forte proportion de grattoirs $(24,5 \%)$ et de denticulés $(35,5 \%)$, importance des éclats à enlèvements irréguliers $(14 \%)$. Ces pièces ne sont pas sans rappeler les types que J. Hinout (1984) a décrit pour le Sauveterrien du Sud du Bassin Parisien. L'industrie laminaire se résume à une lamelle à bord abattu, à une pointe du Tardenois (pointe à base droite) et à quelques pièces allongées 

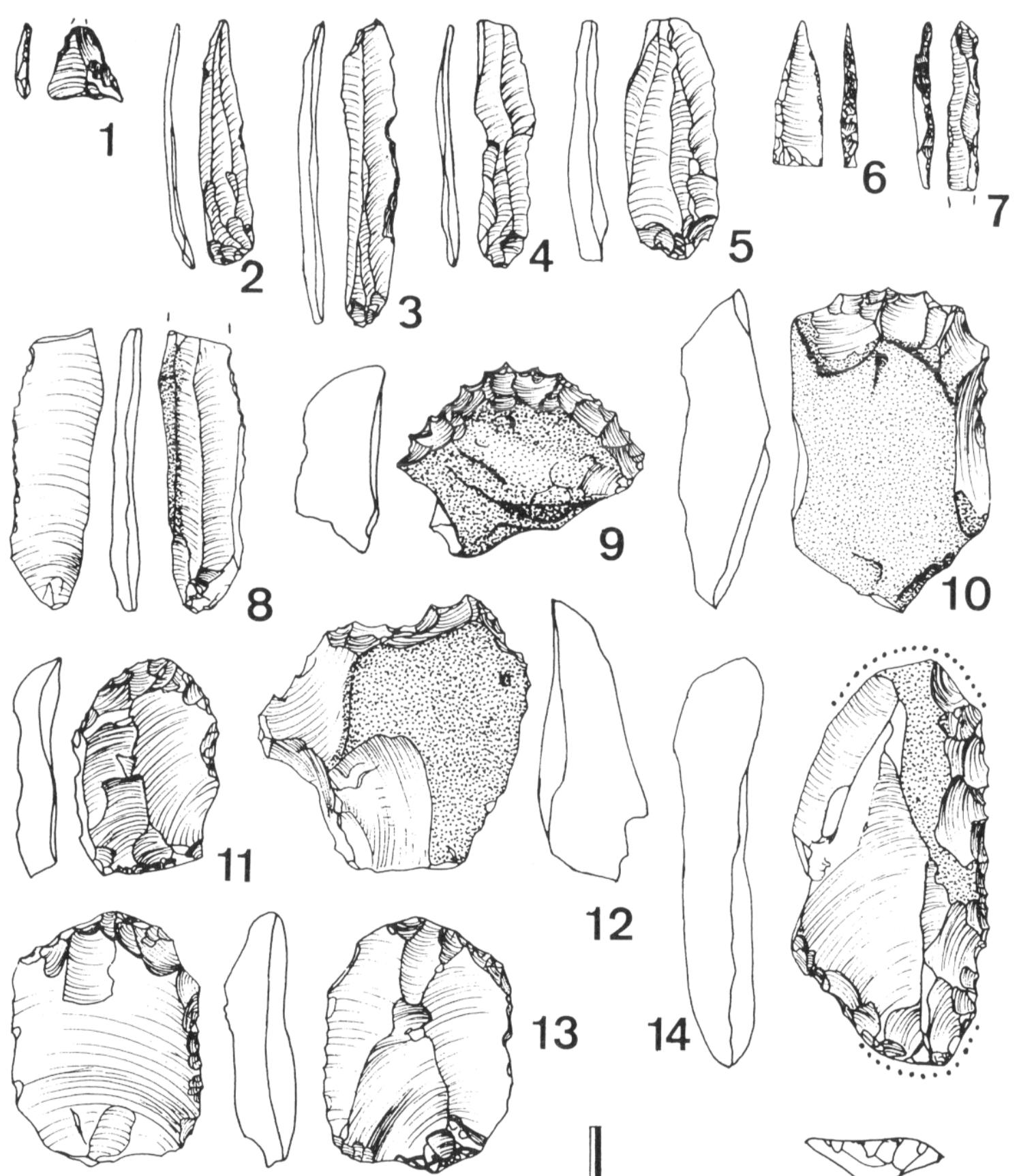

12
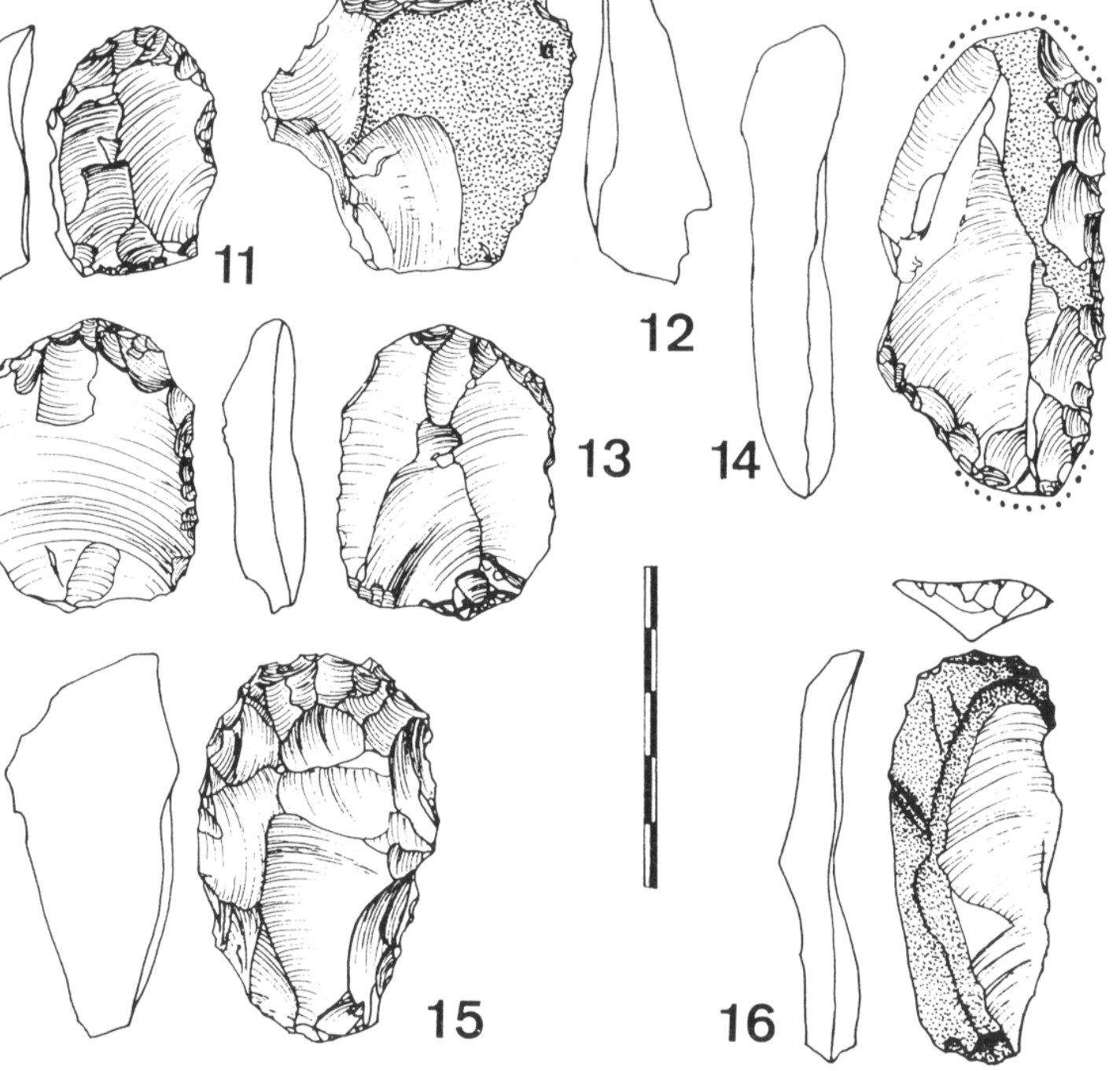

Fig. 4 - Quelques pièces de l'industrie lithique de Noyen. 
retouchées ou utilisées. Ces particularités (majorité des outils sur éclat du fonds commun) sont peut-être à rattacher aux activités menées sur le site : travail du bois, exploitation des produits de la chasse et de la pêche...

L'industrie de la couche 9 sup., bien que très pauvre (au total 30 pièces), se distingue de la précédente par la présence de 23 lames dont certaines rappellent les lamelles Montbani (retouches grignotées des bords dues à une utilisation). La matière première employée est également un silex local. L'utilisation par action transversale sur matière dure végétale (détermination F. Collin, Université de Liège) est attestée pour une grande partie des lames. Le reste est constitué de quelques éclats à enlèvements irréguliers et d'une armature de flèche (trapèze ?). Celle-ci présente une micro-trace linéaire d'impact sans doute due à son utilisation comme pointe de projectile.

Les industries du Mésolithique final et de la transition Mésolithique/Néolithique ancien montrent une évolution importante qui se caractérise par le développement de la méthode de débitage laminaire. Finalement, celle-ci ne croîtra que faiblement (site de Balloy «Les Réaudins »; Augereau et al., sous presse) et disparaitra totalement dès la transition Néolithique ancien régional/Néolithique moyen (site de Châtenay-sur-Seine " Les Pâtures »; Augereau, à paraître). La méthode de débitage la plus simple avec une dominance des grattoirs prévaudra alors, jusqu'à la fin du Néolithique moyen. La question de l'origine culturelle de la technique laminaire, et les raisons de son apparition dans le système techno-économique puis de sa disparition sont actuellement à l'étude.

\section{III.2.2 - Les objets façonnés en matière végétale}

Ils représentent un ensemble unique en France et même en Europe, par son âge (8000 BP : Boréal) et sa diversité :

- 5 nasses et objets de technique comparable,

- un petit récipient en vannerie à double brins cordés serrés,

- une pirogue monoxyle en pin sylvestre (longueur conservée : 4 mètres).

Avant l'étude archéologique détaillée de ces objets se pose le délicat problème de leur conservation. Plusieurs pièces ont déjà été traitées, d'autres attendent une méthode appropriée.

\section{III.2.3 - L'industrie en os et en bois de Cervidés}

L'étude zooarchéologique montre que le spectre d'âge obtenu sur les bois de Cerf du Mésolithique moyen ne correspond en rien à celui que donnent les restes des parties consommées (squelette post-crânien). Cela implique une récolte importante, un transport et une importation sur le site de matière première mais aussi d'objets façonnés en bois de cerf. De fait un certain nombre d'objets finis ont été récoltés et de nombreux bois ou portions de bois montrent des traces de chauffe et d'extraction. Notons que les bois de Chevreuil n'ont pas fait l'objet de la même attention.

Les os longs ont également été exploités durant le Mésolithique : 8 à $9 \%$ des métapodes de Cerf et quelques os d'Aurochs portent des traces d'extraction de matière (souvent par double rainurage). Quelques outils finis sont en cours d'étude.

\section{III.2.4 - Peaux et fibres animales}

L'étude de la fréquence des parties du squelette des Sangliers, des Cerfs et des Chevreuils des horizons mésolithiques montre un déficit voire une absence de $3^{\mathrm{e}}$ phalange. Cela témoigne du prélèvement systématique de la peau sur le lieu de chasse (Cerf, Sanglier) ou au camp de base (Chevreuil). Celle-ci était précieusement conservée puisque ses restes ne figurent pas dans les déchets « domestiques ». Aucun indice de prélèvement de la peau des Aurochs n'apparaît (pas de déficit de $3^{\mathrm{e}}$ phalanges), mais il est probable que les Mésolithiques ne manquaient pas d'utiliser la fourrure des petits carnivores (notamment la Loutre) dont on trouve d'assez nombreux restes dans les déchets alimentaires.

De curieuses traces longitudinales terminées en virgule ont été observées sur plusieurs os longs (radius, métapodes) de Cerf et de Chevreuil. Ils témoignent probablement du prélèvement de ligaments. Ils affectent 3 à $4 \%$ des métapodes de Cerf.

\section{III.3 - L'Homme}

L'Homme n'est pas représenté que par les traces de ses activités. Un certain nombre de restes squelettiques ont été retrouvés dans les chenaux tourbeux. Ils sont assez peu nombreux mais présentent un double intérêt : ils sont les premiers trouvés, pour le Boréal, au nord de la Loire, et plusieurs restes portent des traces de découpe, de même facture que celles portées par les os animaux. La comparaison est en cours.

\section{CONCLUSION}

Par l'état de conservation des vestiges et par la séquence chronologique qu'il offre, Noyen-sur-Seine 
apparaît clairement comme un site clé pour la compréhension du Mésolithique et de la néolithisation du Bassin Parisien. Cela justifiait, s'il en était besoin, la mise en place d'une équipe pluridisciplinaire pour son étude.

Les résultats qui viennent d'être présentés sont pour la plupart inédits. Un important travail de confrontation est en cours dont nous avons tenté de montrer les premiers aboutissements dans les lignes qui précèdent. On peut dès à présent, souligner les grandes étapes interdisciplinaires de ce cheminement.

Les premiers éléments de compréhension stratigraphique ont été obtenus par l'équipe de fouille à qui revenait la lourde responsabilité de démêler l'enchevêtrement des chenaux "entrecroisés ». Elle y fut aidé par les géologues et géomorphologues. Au cours des campagnes de fouilles suivantes, les données palynologiques, radiochronologiques et malacologiques ont aidé à affiner la compréhension chronostratigraphique. Les résultats préliminaires des études de faunes (Poissons, Mammifères) sont venus confirmer les impressions de la fouille. Mais, comme souvent - et il faut le regretter - la plupart des études approfondies n'ont été réellement engagées qu'après la fin de la fouille ce qui interdit tout retour au terrain, surtout dans le cas présent où le site est aujourd'hui remplacé par une gravière.
Les auteurs sont membres du projet 970061 de l'A.T.P. du C.N.R.S. ( Développement d'approches nouvelles en Archéologie »). Muséum national d'Histoire naturelle, Laboratoire d'Anatomie comparée, 55, rue de Buffon, F - 75005 Paris.

Augereau A., Leroyer C. et Tresset A. (sous presse) - La transition Néolithique ancien/Néolithique moyen dans la vallée de la Petite Seine : contribution des études lithiques, zooarchéologiques et palynologiques. Actes $X V I^{e}$ Coll. Interrégional sur le Néolithique (Paris, 1989), Doc. Archéol. Fr.

Dauphin C. (1987) - L'ichtyofaune de Noyen-sur-Seine. Actes $112^{e}$ Congrès nat. Soc. savantes (Lyon, 1987), Protohistoire, pp. 11-32.

Delibrias G., Duperon J., Michel J.-P., Renault-Miskovsky J., Tomasson R. et Van Den Brink S. (1982) - Datations isotopiques, Paléobotanique, Paléontologie et Géologie de dépôts tardiglaciaires et postglaciaires de l'Est du Bassin Parisien (région de Nogent-sur-Seine, Aube). Cahiers Géologiques Université P.-et-M. Curie, Paris, 100, pp. 565-582.

FréCAut R. et PAGNey P. (1983) - Dynamique des climats et de l'écoulement fluvial. Masson éd., Paris, 239 p.

LERETON J.-C. (1974) - Dynamique fluviale, Eyrolles éd. (Collection de la Direction des Études et Recherches d'Électricité de France), Paris, 209 p.

Mordant C. et Mordant D. (1987) - Noyen-sur-Seine, site mésolithique en milieu humide fluviatile. Actes $112^{\circ}$ Congrès nat. Soc. savantes (Lyon, 1987), Protohistoire, pp. 33-52.

Vigne J.-D. et MARINVAL-VIGNE M.-C. (1988) - Quelques réflexions préliminaires sur les Canidés mésolithiques de Noyen-sur-Seine (France) et sur la domestication du chien en Europe occidentale. Archaeozoologia, 2 (1-2), pp. 153-164. 